\title{
The Lead Radius Experiment PREX
}

\author{
Robert Michaels \\ Thomas Jefferson National Accelerator Facility 12000 Jefferson Ave Newport News, VA, USA \\ Submitted to European Journal of Physics, Sept 2006
}

\begin{abstract}
The proposed PREX experiment at Jefferson Lab will measure the parity-violating electroweak asymmetry in the elastic scattering of polarized electrons at an energy of $850 \mathrm{MeV}$ and a scattering angle of $6^{\circ}$. Since the $Z_{0}$ boson couples mainly to neutrons, this asymmetry provides a clean measurement of $R_{n}$ with a projected experimental precision of $\pm 1 \%$. In addition to being a fundamental test of nuclear theory, a precise measurement of $R_{n}$ pins down the density dependence of the symmetry energy of neutron rich nuclear matter which has impacts on neutron star structure, heavy ion collisions, and atomic parity violation experiments.
\end{abstract}

PACS. 20.10.Gv Mass and neutron distributions - 25.30.Bf Elastic electron scattering - 11.30.Er Parity Violation

\section{JLab Copyright}

Notice: Authored by The Southeastern Universities Research Association, Inc. under U.S. DOE Contract No. DE-AC05-84150. The U.S. Government retains a non-ex clusive, paid-up, irrevocable, world-wide license to publish or reproduce this manuscript for U.S. Government purposes.

\section{Introduction}

In a heavy nucleus like ${ }^{208} \mathrm{~Pb}$ the difference between the neutron radius $R_{n}$ and the proton radius $R_{p}$ is believed to be several percent. This neutron skin has not been well established experimentally in stable nuclei. We plan to measure the neutron charge radius $R_{n}$ (i.e. the RMS radius of neutrons in a nucleus) in a clean and model independent way analogous to the classic measurements[1] of the proton radius $R_{p}$ and with unprecedented accuracy as suggested originally by Donnelly, Dubach, and Sick[2].

The experiment measures the parity violating asymmetry in elastic scattering $A=\left(\sigma_{R}-\sigma_{L}\right) /\left(\sigma_{R}+\sigma_{L}\right)$. This asymmetry arises due to the the interference of the $Z^{0}$ boson amplitude of the weak neutral interaction with the photon amplitude. The asymmetry is sensitive mainly to the neutron radius $R_{n}$ because the weak charge of the neutron is much larger than that of the proton. In PWIA, the relationship between the asymmetry and the neutron form factor is given by equation (1)

$$
A_{L R}=\frac{G_{F} Q^{2}}{4 \pi \alpha \sqrt{2}}\left[1-4 \sin ^{2} \theta_{W}-\frac{F_{n}\left(Q^{2}\right)}{F_{p}\left(Q^{2}\right)}\right]
$$

where $G_{F}$ is the Fermi constant, $\alpha=\frac{1}{137}$ is the fine structure constant, $\theta_{W}$ is the Weinberg angle, and $F_{n}\left(Q^{2}\right)$ and $F_{p}\left(Q^{2}\right)$ are the neutron and proton form factor of the nucleus. Thus $A_{L R}$ is approximately proportional to the ratio of neutron to proton form factors. In the above we used PWIA to illustrate. To achieve $1 \%$ accuracy requires corrections for Coulomb distortions, which have been calculated by Horowitz[4].

\section{Physics Impact and Interpretation}

It is remarkable that a single measurement of $R_{n}$ with $1 \%$ accuracy can have such a broad impact on several areas of physics, including neutron star formation and structure[5], atomic parity violation[6,7], nuclear theory[3], and and heavy ion collisions[8].

From a theoretical perspective, the difference between our proposed measurement of $R_{n}$ and the well-measured proton radius $R_{p}$ is determined by fundamental parameters of bulk nuclear matter called the symmetry energy $S_{\nu}$ and the density dependence of the symmetry energy $S_{\nu}^{\prime}$. These parameters are important for other phenomena involving nuclear matter, such as heavy ion collisions. Today, perhaps the most important application is the field of supernovae and the properties of neutron stars. The proposed measurement of $R_{n}$ to $1 \%$ will provide the best determination of the $S_{\nu}$ and $S_{\nu}^{\prime}$ at the density of ordinary nuclear mater. This will provide vital input to the dynamics of stellar explosions and thus have implications for our understanding of how the heavy elements in our world were produced.

The relevant part of the energy of nuclear matter of density $n$ and ratio $x$ of protons to neutrons may be writ- 
ten

$$
E(n, x)=E(n, x=1 / 2)+S_{\nu}(n)\left(1-2 x^{2}\right) .
$$

The connection between $S_{\nu}\left(n_{0}\right)$, where $n_{0}$ is the density of $\mathrm{Pb}$, our measurement, and $R_{n}$ is shown by Furnstahl[9] within the context of realistic nuclear calculations. The model dependence of the relations is small compared to our required sensitivity.

The importance of the symmetry energy $S_{\nu}$ for the formation and structure of neutron stars is given in several review articles[10,11]. A good introduction for the non-specialist by Lattimer and Prakish[12] appeared in Science. Neutron stars are giant nuclei $\sim 13 \mathrm{~km}$ in diameter, fascinating objects for which astronomical data are rapidly improving. Data on neutron stars include radii, masses, luminosity, temperature, and cooling rates [13]. In addition to their intrinsic interest as newly explored natural phenomena, these stars provide "laboratories" to understand the nature of extremely dense matter. For example, do collapsed stars form "exotic" phases of matter, e.g. strange stars or quark stars?

A precise measurement of $R_{n}$ provides a calibration of the equation of state (pressure versus density) of neutron rich nuclear matter, which is an important input for calculating the structure of neutron stars[5]. To rule in or out possible exotic phases of dense matter one needs to combine the high density measurements of neutron stars with low density precision measurements of $R_{n}$ in nuclei. $R_{n}$ also has implications for the crust thickness because the transition density for the liquid-solid phase transition depends sensitively on the neutron skin of ${ }^{208} \mathrm{~Pb}[5]$. The proton fraction of neutron rich matter in beta equilibrium depends on the symmetry energy, which is calibrated by $R_{n}$. A large symmetry energy favors more protons, and if the proton fraction is high enough then the following "URCA" process can cool neutron stars $n \rightarrow p+e^{-}+\bar{\nu}_{e}$ ; $p+e^{-} \rightarrow n+\nu_{e}$ where the $\nu_{e}, \bar{\nu}_{e}$ carry off energy. URCA cooling might explain recent Chandra observations of the neutron star 3C58, a remnant of the supernova seen in the year 1186 that appears to be unexpectedly cold[13]. A neutron skin larger than about $0.2 \mathrm{fm}$ may imply that URCA cooling is possible, while a smaller skin implies it is probably not possible[14].

Heavy ion reactions $[15,16,8]$, including rare isotope beam experiments anticipated from RIA[17], can also pin down the density dependence of the symmetry energy by probing conditions at neutron densities up to 3 times normal nuclear matter density. A combination of our measurement at sub-nuclear densities and the RIA results will provide useful constraints on nuclear theory.

The impact of an accurate $R_{n}$ measurement on atomic parity violation (APV) experiments has been analyzed by Pollock et.al [3],[7]. Knowledge of $R_{n}$ at the $1 \%$ level is needed for interpreting atomic physics measurements of the Weinberg angle at the level of the Standard Model weak radiative corrections. The most accurate ( $1 \%$ in $\left.Q_{\text {weak }}\right)$ measurement of APV was carried out by Wieman and coworkers in atomic cesium ${ }^{133} \mathrm{Cs}[18]$. Such low energy experiments provide powerful constraints on the standard model [19]. More independent tests of APV are needed at the accuracy of the Cesium experiment. An alternative approach which largely cancels the uncertainties due to difficult atomic structure calculations is to measure ratios of APV amplitudes between isotopes [20]. This is being exploited for example in the Berkeley atomic Yb experiment [21]. However, Fortson et.al. [22] pointed out that this technique has an enhanced sensitivity to uncertainties in $R_{n}$. A recent analysis[6] has emphasized the importance of this experiment on the field.

There have been renewed attempts to obtain $R_{n}$ from hadronic data. A major effort has been devoted to the analysis of proton scattering [23-26]. Clark and Kerr [25] arrive at the smallest claimed error on $R_{n}$, less than $1 \%$. Piekarewicz[23] suggests that the error should be at least $3 \%$. Karataglidis et al.[24] suggest that the proton and proposed data are complementary and that both are important. We note that recent data on pion photoproduction from lead and other nuclei [27] have yielded matter radii that are less than the charge radii, implying a negative neutron skin in ${ }^{208} \mathrm{~Pb}$.

The physics interpretation of the experiment can be summarized as follows. From the measured asymmetry one may deduce the weak form factor, which is the Fourier transform of the weak charge density at the momentum transfer of the experiment. One must correct for Coulomb distortions, which has been done accurately by Horowitz[4] and others[28]. The weak charge density can be compared directly to theoretical calculations and this will constrain the density dependence of the symmetry energy. The weak density can be directly applied to atomic parity violation because the observables have approximately the same dependence on nuclear shape. From the weak charge density one can also deduce a neutron density at one $Q^{2}$ by making small corrections for known nucleon form factors. The uncertainty in these corrections for a realistic experiment have been estimated and are small[3].

Finally from a low $Q^{2}$ measurement of the point neutron density one can deduce $R_{n}$. This requires knowledge of the surface thickness to about $25 \%$ to extract $R_{n}$ to $1 \%$. The spread in surface thickness among successful mean field models is much less than $25 \%$, hence we can extract $R_{n}$ with the desired accuracy as shown by Furnstahl[9]. In summary, the physics results of the experiment are the weak charge density, the point neutron density and $R_{n}$.

\section{Experiment}

\subsection{Experimental Overview}

The ${ }^{208} \mathrm{~Pb}$ experiment was proposed for a beam energy of $850 \mathrm{MeV}$ and a $6^{\circ}$ scattering angle in Hall $\mathrm{A}$ at Jefferson Lab. The two Hall A 3.7 msr spectrometer systems supplemented by septum magnets focus elastically scattered electrons onto total-absorption detectors in their focal planes. A $50 \mu \mathrm{A}, 80 \%$ polarized beam with a $30 \mathrm{~Hz}$ helicity reversal will scatter from a foil of lead which is sandwiched between sheets of diamond to improve the thermal characteristics. Ratios of detected flux to beam 
Table 1. Rate and Asymmetry for ${ }^{208} \mathrm{~Pb}$

\begin{tabular}{|r|r|}
\hline Measured Asymmetry $\left(p_{e} A\right)$ & $0.51 \mathrm{ppm}$ \\
Beam Energy & $850 \mathrm{MeV}$ \\
Beam Current & $50 \mu \mathrm{A}$ \\
Required Statistical Accuracy & $3 \%$ \\
Energy Cut (due to detector) & $4 \mathrm{MeV}$ \\
Detected Rate (ea spectrometer) & $860 \mathrm{MHz}$ \\
Running Time & 680 hours \\
\hline
\end{tabular}

current integrated in the helicity period are formed, and the parity-violating asymmetry in these ratios computed from the helicity-correlated difference divided by the sum: $\mathrm{A}=\left(\sigma_{R}-\sigma_{L}\right) /\left(\sigma_{R}+\sigma_{L}\right)$, where $\sigma_{R(L)}$ is the ratio for $\operatorname{right}(R)$ an $\operatorname{left}(L)$ handed electrons. Separate studies at lower rates are required to measure backgrounds, acceptance, and $Q^{2}$. Polarization is measured once a day by a Møller polarimeter, and monitored continuously with the Compton polarimeter.

Table 1 shows the rates, asymmetries, and running times for the ${ }^{208} \mathrm{~Pb}$ parity experiment proposal, the results averaged over the acceptance.

\subsection{Technical Issues}

For the ${ }^{208} \mathrm{~Pb}$ experiment the asymmetry of $0.5 \mathrm{ppm}$ must be measured to $3 \%$ accuracy. Both the absolute error (15 $\mathrm{ppb})$ and the relative accuracy $(3 \%)$ are challenging to achieve. The main issues affecting the absolute error are the control of false asymmetries associated with helicity correlations in beam parameters such as intensity, energy, and position. The main issues affecting the relative error are the beam polarimetry and measurement of $Q^{2}$. In addition, we need a lead target that can withstand a high beam current.

\subsection{Helicity Correlated Systematics}

To evaluate the systematic error we need to know the helicity correlated differences as well as our sensitivity to these which are measured online by modulation of the parameters. During the 2005 HAPPEX run we have made tremendous progress in controlling helicity correlated systematics, see fig 1 which shows 1-month averages of $\sim 1$ nanometer in helicity correlated position differences. This accomplishment is due to a long-term effort to improve the setup of the polarized source and accelerator. For the polarized source we have a well-developed model for controlling the laser systematics, which allows us to minimize helicity correlations in the laser beam used to produce polarized electrons. For the accelerator, progress in understanding the betatron matching has helped achieve maximum dampening of position differences, while improved understanding of beam tuning has provided both excellent signal-to-noise ratios in the Compton polarimeter and good phase advances along our beamline that permit us to simultaneously have good measurements to the sensitivities of each of the independent beam parameters. For ${ }^{208} \mathrm{~Pb}$ we will maintain position differences to less than 1 $\mathrm{nm}$ with an accuracy of $0.1 \mathrm{~nm}$ averaged over a 1 month run. The charge asymmetry will be maintained to less than $100 \mathrm{ppb}$ with an accuracy of $10 \mathrm{ppb}$.

\subsection{Lead Target Tests}

We have built a high power lead target which will be stable at 40 Watt for a $50 \mu \mathrm{A}$ beam. Successful beam tests at $80 \mu \mathrm{A}$ show that we have a good target design. Recently we have performed low-intensity tests at $1.1 \mathrm{GeV}$, which is approximately the energy of this proposal. These tests checked the rates, backgrounds, resolution, sensitivity to beam parameters (slopes), and width of asymmetry.

\subsection{Noise}

The pulse-to-pulse noise in the lead experiment is 140 ppm. All other noises must be small compared to 140 ppm in order for counting statistics to dominate our errors and to keep the running time minimal. It will be important to avoid long cable runs for our detectors, and therefore we have installed a distributed DAQ system during HAPPEX-2, in which the DAQ crates are situated near the detectors. For understanding the noise, beam systematics, and possible target boiling effects, we have implemented a new luminosity monitor which was used during HAPPEX. New 18-bit ADCs are being fabricated at JLab for use in upcoming parity experiments which will improve our pedestal noise.

\subsection{Precision Polarimetry}

Improvements in polarimetry are of vital importance for the Jefferson Lab parity violation program. For this proposal, the polarization must be measured to $1 \%$ preferably, or at least $2 \%$. With a polarization accuracy of $1 \%(2 \%)$ we can extract $R_{n}$ to $1 \%(1.2 \%)$ respectively. Our plan is to use a combination of upgraded Compton polarimeter and upgraded Møller polarimeter.

The best accuracy obtained so far with the Compton polarimeter is $1.5 \%$ total relative error within 40 minutes for a beam energy of $4.5 \mathrm{GeV}$. We plan to implement a green laser to improve the figure of merit at $850 \mathrm{MeV}$. This brings the mean asymmetry to $0.65 \%$. At the Compton edge the photon energy is $26 \mathrm{MeV}$ and the associated scattered electron is $6 \mathrm{~mm}$ above the primary beam at the location of the electron detector. Assuming the same laser power of $1.5 \mathrm{~kW}$ at the Compton interaction point, a $1 \%$ statistical accuracy is achieved within 16 hours. The laser and much of the associated optics equipment has been obtained and is undergoing test. In addition to the laser, we plan on using a new crystal for the photon detector.

We will compare Møller to Compton at $5 \mu \mathrm{A}$ and 6 $\mathrm{GeV}$ to give a clear comparison at the $1 \%$ level. 

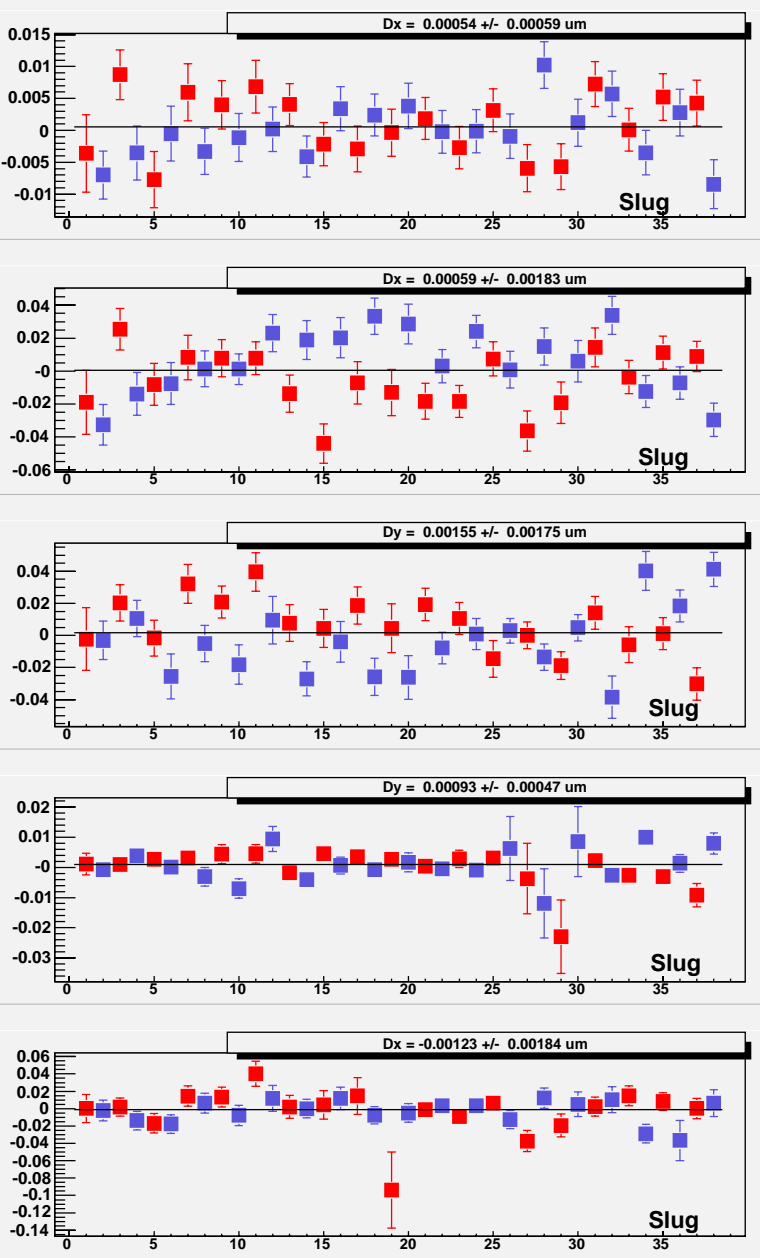

Fig. 1. Helicity correlated beam monitor differences $(\mu \mathrm{m})$ versus slug number (1 "slug" 1 day of running) for HAPPEX-2. The top four plots are $\mathrm{X}$ and $\mathrm{Y}$ monitors near the target, and the bottom plot is a monitor in the dispersive section of the magnet ARC leading into the hall, providing a relative energy monitor. The averages over a month of running are $\sim 1$ nanometer.

\section{Summary and Outlook}

Measurement of $R_{n}$ will have a fundamental impact on nuclear physics and its applications to neutron rich matter. The neutron skin is a qualitative feature of heavy nuclei which has never been cleanly observed. These experiments require state-of-the-art control of systematic errors. The technological developments will help drive other facets of the Jefferson Lab parity program, such as $Q_{\text {weak }}$ which has similar demands.

The HAPPEX run in 2004 and 2005 have demonstrated excellent control of helicity correlated beam parameters. An upgrade of the Compton polarimeter, necessary to achieve $1 \%$ accuracy at $850 \mathrm{MeV}$, is underway.

\section{References}

1. H. de Vries, C. W. de Jager, and C. de Vries, Atomic and Nuclear Data Tables, 36, 495 (1987).

2. T.W. Donnelly, J. Dubach, and I. Sick, Nucl. Phys. A503, 589 (1989).

3. C. J. Horowitz, S. J. Pollock, P. A. Souder, and R. W. Michaels, Phys. Rev. C 63, 025501, (2001).

4. C. J. Horowitz, Phys. Rev. C 57, 3430 (1998).

5. C. J. Horowitz, J. Piekarweicz, Phys. Rev. Lett 86 5647 (2001). C. J. Horowitz, J. Piekarewicz, Phys. Rev. C64, 062802 (2001).C. J. Horowitz, J. Piekarewicz, atro-ph/0201113 (2002). J. Carriere, C.J. Horowitz, J. Piekarewicz nucl-th/0211015 (2002).

6. T. Sil, M. Centelles, X. Vinas and J. Piekarewicz, Phys. Rev. C 71, 045502 (2005) [arXiv:nucl-th/0501014].

7. S.J. Pollock, E.N. Fortson, and L. Wilets Phys. Rev. C 46 2587 (1992).

8. A. W. Steiner and B. A. Li, Phys. Rev. C 72, 041601 (2005) [arXiv:nucl-th/0505051].

9. R. J. Furnstahl, Nucl. Phys. A706, 85 (2002).

10. A. W. Steiner, M. Prakash, J. M. Lattimer and P. J. Ellis, Phys. Rept. 411, 325 (2005) [arXiv:nucl-th/0410066].

11. J. M. Lattimer and M. Prakash, Phys. Rept. 333, 121 (2000) [arXiv:astro-ph/0002203]

12. J.M. Lattimer, M. Prakash, Science 304 (2004) 536.

13. New York Times, April 11, 2002 edition; P. Slane, D. Helfand, S. Murray, astro-ph/0204151; G.G. Pavolv, O.Y. Kargaltzev, D. Sanwal, and G.P. Garmire, ApJ 554, L189 (2001); J.P. Halpern and F.Y.H. Wang, ApJ. 447, 905 (1997); M. Colpi, U. Geppert, D. Page, and A. Possenti, ApJ. 553, 382 (2001); D. G. Yakovlev et.al. astro$\mathrm{ph} / 0204233$.

14. J. Piekarewicz, nucl-th/0207067 (2002).

15. A. Ono, P. Danielewicz, W. A. Friedman, W. G. Lynch and M. B. Tsang, Phys. Rev. C 70, 041604 (2004) [arXiv:nuclth/0409027].

16. D. V. Shetty et al., Phys. Rev. C 70, 011601 (2004) [arXiv:nucl-ex/0406008].

17. B. A. Li, Phys. Rev. C 69, 034614 (2004) [arXiv:nuclth/0312025].

18. C. S. Wood et.al Science 275, 1759, (1997) S.C. Bennet and C.E. Wieman, Phys. Rev. Lett. 82, 2484 (1999).

19. M.-A. Bouchiat and C. Bouchiat, Rep. Prog. Phys. 60, 1351 (1997).

20. A. Derevianko and S.G. Porsev, physics/0112035 (submitted to Phys. Rev. A) (2002).

21. D. DeMille, Phys. Rev. Lett 74, 4165 (1995), C.J. Bowers, et.al Phys. Rev. A53,(5), 3103 (1996); Phys. Rev. A 59, 5 (1999)

22. E.N. Fortson, Y. Pang, and L. Wilets, Phys. Rev. Lett. 65, 2857 (1990).

23. J. Piekarewicz and S. P. Weppner, arXiv:nucl-th/0509019.

24. S. Karataglidis, K. Amos, B. A. Brown and P. K. Deb, Phys. Rev. C 65, 044306 (2002) [arXiv:nucl-th/0111020].

25. B. C. Clark, L. J. Kerr and S. Hama, Phys. Rev. C 67, 054605 (2003) [arXiv:nucl-th/0209052].

26. M. Dupuis, S. Karataglidis, E. Bauge, J. P. Delaroche and D. Gogny, arXiv:nucl-th/0506077.

27. B. Krusche, arXiv:nucl-ex/0509003 Sept 2005

28. See refs in [3], Relativistic Optical Code RUNT, E.D. Cooper, Los Alamos preprint nucl-th/9911024, D. Vretenar et al., and calculations by B.C. Clark et al.. 
29. K. A. Aniol et al., Phys. Rev. Lett. 821096 (1999). K. A. Aniol, et al., Phys. Rev. C 69, 065501 (2004). K. A. Aniol, et al., nucl-ex/0506010. K. A. Aniol, et al., nucl-ex/0506011.

30. P.L Anthony, et.al. Phys.Rev.Lett.95:081601,2005.

31. T. Holmstrom, R. Feuerbach, R. Michaels, " $Q^{2}$ for HAPPEX-2" technical report http://hallaweb.jlab.org/experiment/HAPPEX/docs/qsq_2004.ps

32. M. Hauger et.al. Nucl. Instrum. Meth. A462 382 (2001). 